\title{
Echocardiographic Patterns of Myocardial Fibrosis in Hypertensive Patients: Endomyocardial Biopsy Versus Ultrasonic Tissue Characterization
}

\author{
M. Ciulla, R. Paliotti, D. B. Hess, E. Tjahja, S. E. Campbell, F. Magrini, and K. T. Weber, \\ Milan and Cagliari, Italy, and Columbia, Missouri
}

\begin{abstract}
Echocardiographic image texture has been demonstrated to reflect the physical properties of the tissue under examination. To evaluate the role of collagen in determining the echo pattern of the left ventricular wall, we studied nine hypertensive patients with left ventricular hypertrophy (left ventricular mass index $>$ $125 \mathrm{gm} / \mathrm{m}^{2}$ ) and biopsy-proven different degrees of myocardial fibrosis by analyzing the echocardiographic examinations performed before the biopsy. Myocardial tissue was sampled under fluoroscopy and two-dimensional echo guidance in the interventricular septum. Collagen volume fraction (CVF; normal range up to $2 \%$ ) was taken as an index of fibrosis. The echo patterns were assessed by analyzing standard two-dimensional parasternal long-axis echocardiograms recorded on videotape. Images were color-coded at 256 levels $(0=$ yellow, $256=$ black) and digitized off-line onto a personal computer. The region of analysis was set using a selection tool $(20 \times 10 \mathrm{~mm})$ in the general area of septum where the specimen was taken. For each selection a color-level histogram, representing the frequency
\end{abstract}

$\mathrm{T}$ characterization with ultrasonography represents an extension of conventional echocardiography for the evaluation of myocardial physical properties. ${ }^{1}$ The conventional echo image depicts at a low resolution the interaction between ultrasonographic waves and the singular components that constitute the tissue under study. Associated with the hypothesis that alteration in the echo image pattern is due to alterations in tissue structure, ${ }^{2}$ various approaches have been used to quantitatively define the pathophysio-

From the Centro di Fisiologia Clinica e Ipertensionè, Ospedale Maggiore, Università di Milano, Italy; Cattedra di Medicina Interna, C.L.O., Università di Cagliari, Italy; and the University of Missouri-Columbia.

Reprint requests: Michele Ciulla, MD, Centro di Fisiologia Clinica e Ipertensione, Università di Milano, Ospedale Policlinico, Via F. Sforza, 35, 20122 Milano, Italy; email: img.lab@imizucca.csi. unimi.it.

Copyright $(1997$ by the American Society of Echocardiography. 0894-7317/97\$5.00+0 27/1/80029 distribution, was derived with estimates of the average pixel intensity (mCS), skewness (SK), kurtosis (K), and the broad band $(\mathrm{Bb})$ of the echoes about the distribution. Echo-derived parameters in each patient were compared with corresponding CVF values. CVF was out of range in all patients, ranging from $2.6 \%$ to $7.6 \%$ (mean $4.3 \% \pm 1.6 \%$ ). No correlation was found between CVF and mCS, whereas a significant correlation was found at end diastole between CVF and the parameters describing histogram morphology, respectively, SK $(r=0.73), \mathrm{K}(r=0.69), \mathrm{Bb}(r=0.72)$. These findings for the first time demonstrate in vivo in hypertensive patients with left ventricular hypertrophy an agreement between echo amplitude and histologically assessed collagen volume. Thus in our studied patients collagen content appears to be the major determinant of regional echo intensity, its increase resulting in a significant and progressive wider asymmetrical left shift (yellow) of the color histogram. (J Am Soc Echocardiogr 1997;10:657-64.)

logic state of cardiac muscle with ultrasonography. ${ }^{3}$ Methods for ultrasonic characterization have included gray-scale or backscatter analysis of returning echoes that have been employed in several cardiac disorders. ${ }^{4-6}$

The importance of fibrosis in mediating a pathologic structural remodeling of the hypertensive heart has long been recognized. A marked increase in fibrillar collagen is present in the hypertensive hypertrophied left ventricle and includes the interventricular septum..$^{7,8}$ There is little doubt that collagen deposition, because of its marked acoustic impedance, has an important influence on the echo texture image from the myocardium. ${ }^{9}$ Quantitative analysis of the ultrasound signals from myocardium has demonstrated increased sensitivity for detection of collagen accumulation $^{10,11}$; however, the specificity of changes in ultrasonic reflectivity for the diagnosis of myocardial fibrosis is still uncertain.

We hypothesize that myocardial fibrosis is a major 
Table 1 Patient Population and Myocardial Fibrosis

\begin{tabular}{|c|c|c|c|c|c|c|c|}
\hline No. & Age/Sex & Echo diagnosis & rvs & LVMI & $\mathbf{E} / \mathbf{A}$ & CVF & Histologic findings \\
\hline 1 & $61 / \mathrm{F}$ & cLVH-IAE & 14 & 125.15 & 1.25 & 4.2 & $\begin{array}{l}\text { Focal scars } \\
\text { Endocardial fibrosis }\end{array}$ \\
\hline 2 & $66 / M$ & cLVH-LAE-DD & 12 & 145.85 & 0.74 & 3.8 & $\begin{array}{l}\text { Focal scars } \\
\text { Endocardial fibrosis }\end{array}$ \\
\hline 3 & $40 / \mathrm{F}$ & cLVH & 12 & 206.79 & 1.00 & 2.6 & $\begin{array}{l}\text { Focal scars; endomysial fibrosis } \\
\text { Perivascular collagen }\end{array}$ \\
\hline 4 & $68 / \mathrm{F}$ & aLVH-DD & 16 & 155.93 & 0.72 & 4.9 & $\begin{array}{l}\text { Scars and perivascular fibrosis } \\
\text { Endocardial fibrosis }\end{array}$ \\
\hline 5 & $73 / \mathrm{M}$ & cLVH-DD & 13 & 138.03 & 0.74 & 3.2 & Focal scars \\
\hline 6 & $67 / \mathrm{M}$ & CLVH-DD & 13 & 135.95 & 0.91 & 6.3 & $\begin{array}{l}\text { Interstital fibrosis } \\
\text { Focal scars }\end{array}$ \\
\hline 7 & $76 / \mathrm{M}$ & $\mathrm{cLVH}-\mathrm{MI}$ & 16 & 194.39 & $3.59 *$ & 3.2 & $\begin{array}{l}\text { Interstitial fibrosis; focal scars } \\
\text { Endocardial fibrosis }\end{array}$ \\
\hline 8 & $75 / \mathrm{F}$ & cLVH-DD & 16 & 182.03 & 0.71 & 3.3 & Little interstitial and perivascular fibrosis \\
\hline 9 & $65 / M$ & cLVH-DD & 13 & 129.60 & 0.77 & 7.6 & $\begin{array}{l}\text { Scars; interstitial fibrosis } \\
\text { Endocardial fibrosis }\end{array}$ \\
\hline
\end{tabular}

$I V S$, Interventricular septum $(\mathrm{mm}) ; L V M I$, left ventricular mass index $\left(\mathrm{gm} / \mathrm{m}^{2}\right) ; E / A$, ratio of early/late transnitral filling velocities; CVF, collagen volume fraction (\%); $c L V H$, concentric left ventricular hypertrophy; $L A E$, left atrial enlargement; $D D$, diastolic disfunction; aLVH, asymmetric left ventricular hypertrophy; $M I$, mitral insufficiency.

*Pseudo-normalization of $\mathrm{E} / \mathrm{A}$ ratio.

determinant of echo texture and designed this study in attempt to define echocardiographic indices of myocardial fibrosis. We compared the results of quantitative echo intensity analysis with morphometric analysis of myocardial fibrosis in endomyocardial tissue obtained by transvenous biopsy in hypertensive patients with left ventricular hypertrophy proved by means of echocardiography.

\section{METHODS}

\section{Patient Selection}

We studied nine patients, five men and four women (ages 40 to 76 , mean $66 \pm 11.8$ ), each with a long-standing history of hypertension ( $>10$ years) with left ventricular mass normalized for body surface (left ventricular mass index [LVMI]) calculated according to the Penn Convention $^{12}$ of more than $125 \mathrm{gm} / \mathrm{m}^{2}$ and biopsy-proven myocardial fibrosis of varying degrees. Two patients showed a normal trans-mitral flow; six patients exhibited Doppler echocardiographic findings of left ventricular dysfunction (transmitral $\mathrm{E} / \mathrm{A}$ ratio $<1$ ), and in one patient pseudonormalization with a reversed $\mathrm{E} / \mathrm{A}$ ratio caused by moderate mitral regurgitation was found. The clinical and diagnostic profiles of these patients are summarized in Table 1. A control group of nine normal subjects with normal LVMI $\left(85.6 \pm 23.1 \mathrm{gm} / \mathrm{m}^{2}\right)$ and trans-mitral flow that did not undergo the biopsy procedure was also studied to calibrate the echo texture analysis.

The protocol was approved by the ethical committee of the hospital. All patients gave informed consent for the study.

\section{Endomyocardial Biopsy and Collagen Volume Fraction Determination}

Percutaneous transvenous biopsies from the interventricular septum were performed on all patients. Myocardial tissue was sampled under fluoroscopy and two-dimensional echo guidance in the mid to apical area of the interventricular septum. Analysis of formalin-fixed, paraffin-embedded tissue sections from septal biopsy specimens was performed by means of light microscopy. According to a previously described procedure, ${ }^{7}$ collagen volume fraction (CVF, normal range up to $2 \%$ ), which included scars defined as microscopically discernible areas of focal collagen accumulation that replaced lost cardiac myocytes, was determined with an image analysis system (Quantimet 520; Leica Inc., Deerfield, Ill.). Tissue sections were cut at $5 \mu \mathrm{m}$ thickness, stained with collagen-specific picrosirius red (Sirius Red F3BA in aqueous picric acid), and were analyzed by using normally transmitted light and $540 \mathrm{~nm}$ bandpass filter for contrast enhancement. Sections were quantitated at $80 \times$ magnification.

\section{Echocardiographic Studies}

We performed two-dimensional echocardiography and simultaneous electrocardiographic tracings with a strict time lapse before the diagnostic endomyocardial biopsy, using a standard electronic scanner operating at $3.5 \mathrm{MHz}, 256$ gray levels (Acuson I28XP; Acuson Inc., Mountain View, Calif.). The echocardiograms were obtained with the patients in a let lateral decubitus position. Standard parasternal long-axis echocardiographic views were obtained and images were recorded on videotapes (S-VHS format, system NTSC) for subsequent analysis. The take-off point for the videotape recorder was at the linear output of the digital scan converter of 


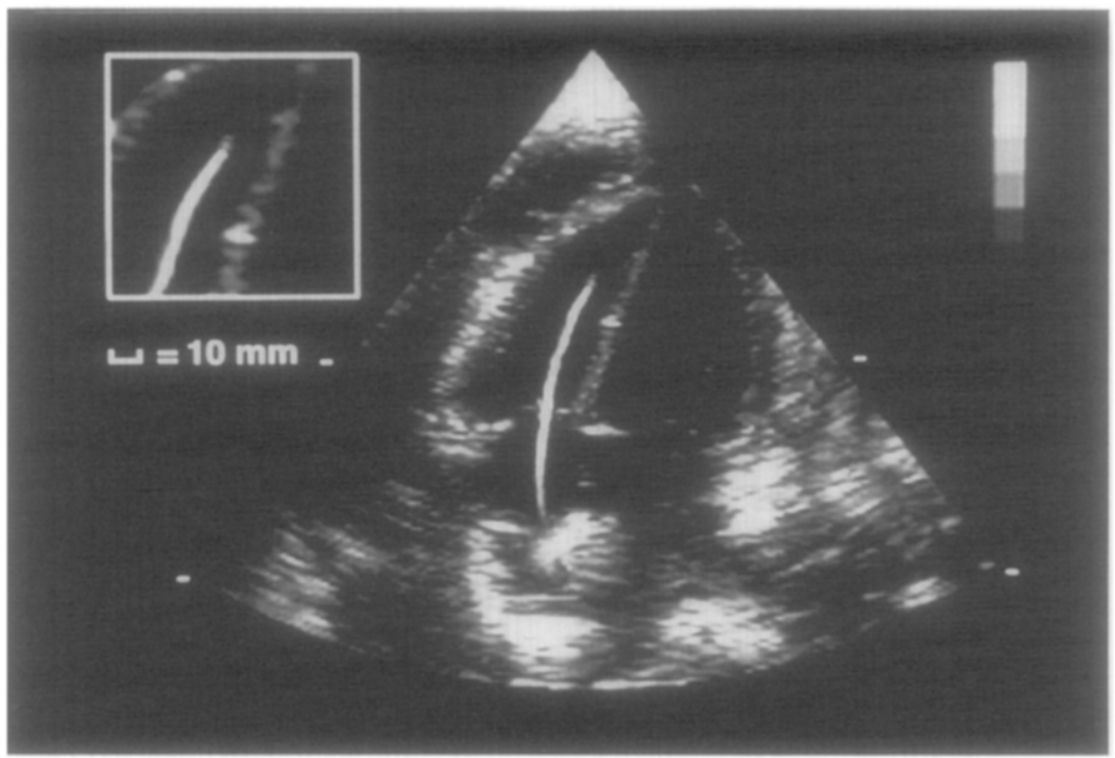

Figure 1 Echo images obtained during the biopsy procedure showing the tip of the bioptome piercing the endocardium.

the echo instrument. The focus was set to the mid-apex interventricular septum that was kept perpendicular to the main echo beam.

Gain settings and gain compensation profiles were adjusted to obtain apparently uniform myocardial brightness throughout the echocardiogram in each subject, and no reject was used. In this study we presumed that the attenuation of chest wall remains constant throughout the cardiac cycle.

\section{Digitalization}

Two representative subsequent sinus rhythm cardiac cycles in each subject were selected and digitized off-line from the videotape onto a personal computer (Macintosh Power PC 8100av, RAM 16 MB, HD 500 MB; Apple Computer, Inc., Cupertino, Calif.) by using the built-in video digitizer card operating at 30 frames $/ \mathrm{sec}, 8 \mathrm{bit} /$ pixel, 256 colors coded on a NTSC standard pixel matrix $(640 \times 480$ pixels $)$. To compensatc for heart rate variability, nine single frames in each subject corresponding to visual cardiac events (opening and closing of aortic and mitral valve) were frozen, labeled as a percentage of the cardiac cycle, and stored onto the hard disk driver of the computer in standard TIFF (tagged image file format) ${ }^{13}$ JPEG compressed computer format (compression ratio $5: 1)^{14}$ for the subsequent analysis.

The region for the analysis was positioned by means of a rectangular selection tool $(10 \times 20 \mathrm{~mm})$ in the mid-apex septum in the general area where the specimen was taken, using as reference the echo images obtained during the biopsy procedure showing the tip of the bioptome piercing the endocardium (Figure 1).

The selected region was kept in each patient over the same myocardial area during the display of the frames by the use of anatomic landmarks in the image, including as much of the transmural myocardium, while avoiding artifacts or areas of echo dropout. In the control group comparative areas were selected.

\section{Image Analysis}

The echo-texture characteristics were determined in the rectangular selection in each selected frame corresponding to two consecutive cardiac cycles as follows. To obtain a better visualization of the structures under study, color scale was set at 256 colors $(0=$ yellow, $128=$ magenta, $256=$ black) re-coding the original 256 echo gray scale $(0=$ white, $256=$ black $)$.

\section{Mean Color Scale Value During Cardiac Cycle}

The mean color scale value ( $\mathrm{mCS}$ ) and the standard deviation ( \pm SD) were assessed in both groups by using an imageprocessing software developed in our laboratory and implemented on the video digitizer manager specifications (Perceptics Corp., Knoxville, Tenn.). To avoid the effects on image texture caused by the characteristics of the ultrasound image system, all values obtained were nornalized for blood echoreflectivity (black $=256$ ) assessed along the same axis inside the left ventricle (Figure 2). The mean color value describes the overall brightness/darkness of the image; the standard deviation, the overall contrast of the image.

\section{Histogram Study}

For each selection a color histogram representing the pixel color-level frequency distribution was derived with estimates of skewness (SK), which demonstrates asymmetry of the distribution, kurtosis $(K)$, which depicts the peakedness 


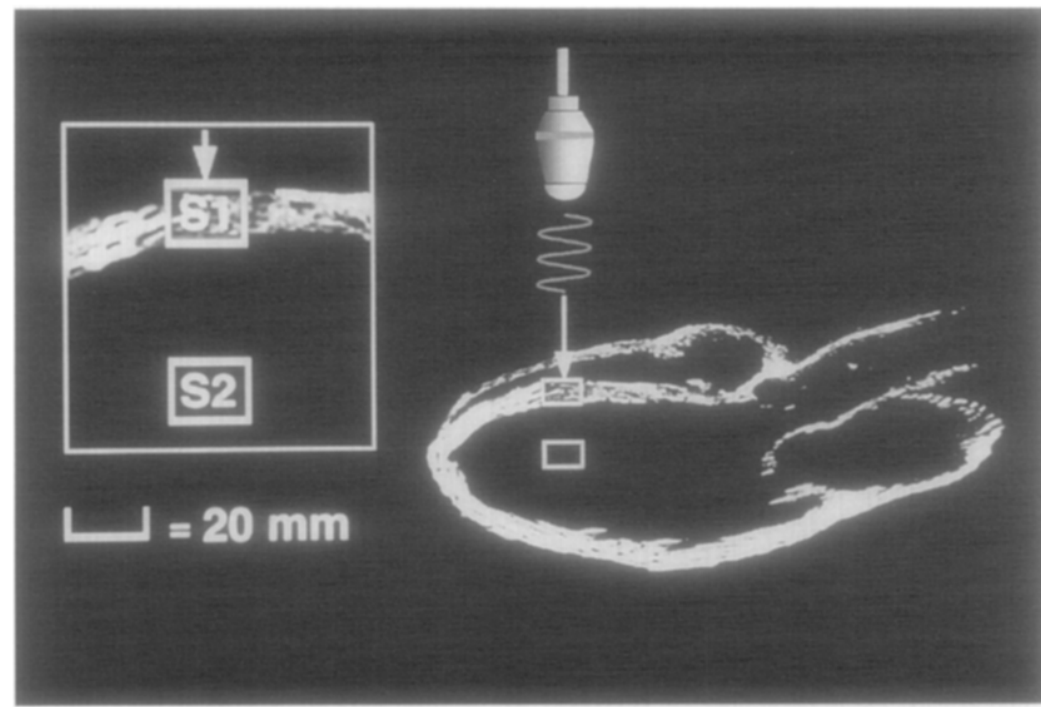

Figure 2 Schematic diagram of a long-axis view of the heart illustrating the region of interest (SI) in the mid-apex interventricular septum from which the measurements were taken. Values obtained were normalized for blood echoreflectivity (black $=256$ ) assessed along the same axis inside the left ventricle (S2).

of the curve, and the broad band ( $\mathrm{Bb}$ ), which indicates the spread of the echoes about the distribution.

\section{Statistical Analysis}

Data were analyzed by means of a computer statistical software (SPSS-Rel 6.1.1; SPSS Inc., Chicago, Ill.). Values obtained are reported as mean $\pm \mathrm{SD}$; the group differences in mean pixel intensity between controls and hypertensives with and without Doppler findings of left ventricular diastolic dysfunction were tested for significance using the two-way analysis of variance applying the Bonferroni correction for multiple comparison.

The relation between collagen content and echoreflectivity-derived parameters in patients with biopsy-studied patients was expressed in terms of linear regression analysis. A probability $(p)$ value less than 0.05 was considered significant.

Interobserver reproducibility was evaluated comparing the measurements made by observer 1 with those of observer 2; intraobserver reproducibility was evaluated in the same way, comparing the data derived by one observer on two different days. Results are reported as coefficient of variation.

\section{RESULTS}

The bioptic procedure was well tolerated in all studied patients. No adverse reactions or complications occurred.

\section{Biopsy Data}

Histologic patterns of myocardial fibrosis are reported in Table 1. According to previous studies in normal human tissue, where collagen volume fraction was found to be $1.6 \pm 0.9,{ }^{15}$ collagen tissue volume fraction was increased in biopsy tissue ranging from 2.6 to $7.6 \%$ (mean $4.3 \% \pm 1.6 \%$ ).

\section{Echoreflectivity results}

As previously reported, ${ }^{16}$ mean color scale value, which represents the median pixel intensity directly related to the lightness of the image, showed a cyclic profile according to cardiac phases (systolic to diastolic). Changes were in phase with a similar curve profile in control subjects and in patients with normal diastolic function, and in patients with diastolic dysfunction smooth changes with an out-of-phase curve profile were found (Figure 3); differences observed in absolute values of mCS between the three groups were not statistically significant.

Histogram shapes, representing the pixel colorlevel frequency distribution, were substantially different in each patient that underwent the biopsy (Figure 4), showing a progressive asymmetrical left shift of the color histogram (corresponding to yellow in our scale) with the increase of collagen content (Figure 5 ); mean values of $\mathrm{SK}, \mathrm{K}$, and $\mathrm{Bb}$ at end diastole were significantly different if compared with corresponding values obtained in control patients (Table 2 ). 


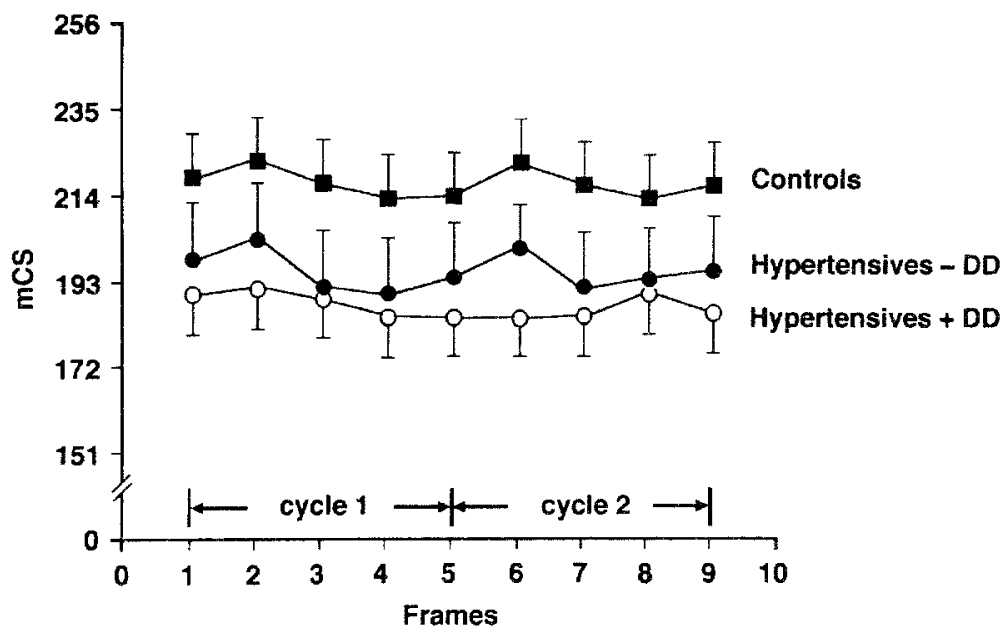

Figure 3 Cyclic changes of mean color scale values $(m C S)$ during two cardiac cycles in controls $(n=9)$ and in hypertensive patients with $(n=6)$ and without diastolic dysfunction $(n=3)$, respectively, $+D D$ and $-D D$.

\section{Comparison of Methods}

No parameter related to the mean pixel intensity during cardiac cycle discriminates different levels of fibrosis. On the other hand, at end diastole a significant correlation was found between CVF and parameters describing the shape of the color histogram, respectively: SK $(r=0.73), \mathrm{K}(r=0.69), \mathrm{Bb}(r=$ 0.72 ) (Figure 6 ). The coefficient of variation between measurements made was less then $3 \%$ (inter- and intra-observer, respectively, $\pm 3 \%$ and $\pm 2 \%$ ).

\section{DISCUSSION}

The unique echo pattern that various tissue display reflects to some extent their collagenous content that is considered the major source of echogenicity in tissues. 9,11 Several studies in vitro and in vivo in different pathological conditions involving fibrosis such as cardiomyopathy ${ }^{1,17-19}$ or myocardial infarction $^{20}$ have shown a promising correlation between echoreflectivity and histologically or biochemically assessed collagen content suggesting the possibility of making non invasive ultrasonic quantification of collagen accumulation in tissues.

The present study, though it reports a limited number of observations, demonstrates a direct correlation between histologically assessed collagen in biopsy tissue and pixel-color level frequency distribution in hypertensive patients with left ventricular hypertrophy.

All patients studied showed an excessive collagen accumulation: these data are consistent with previous evidence of an increased collagen content in autopsied hearts of patients with hypertension and left ventricular hypertrophy. ${ }^{15,21}$ Other authors, assuming echoreflectivity as an index of fibrosis and without bioptic control, have found a normal pattern of quantitatively assessed ultrasonic backscatter in mild to moderate hypertensive patients with different degrees of left ventricular hypertrophy, suggesting a balanced increase of the myocyte and connective tissue components of the myocardium. ${ }^{22}$ The different acoustic patterns we observed are not in contrast with these data, but reflect the different structural and architectural characteristics of the studied hearts: our patients were older and had a longer history of hypertension if compared with other studied hypertensive patients.

With the increase in collagen content we report a progressive wider asymmetrical left shift of the color histogram. The mean value of the color scale that is directly related to the lightness of the image was not relevant to collagen content, while the spread-out of echoes and the shape of the distribution showed a significant correlation factor. According to the physical behavior of ultrasounds, we hypothesize that the increase in collagen network increases the casual constructive and destructive interactions of echoes within the tissue; in a simplified model it is possible to consider collagen fibers acting as diffuse reflectors inside the tissue returning echoes in all directions producing a wider color spectrum.

Since ultrasounds are sensitive to the anisotropic properties of tissues, we cannot exclude that myocardial fiber disarray, observed in hypertrophic cardiomyopathy, may be another important contributing factor in the genesis of echoreflectivity. 


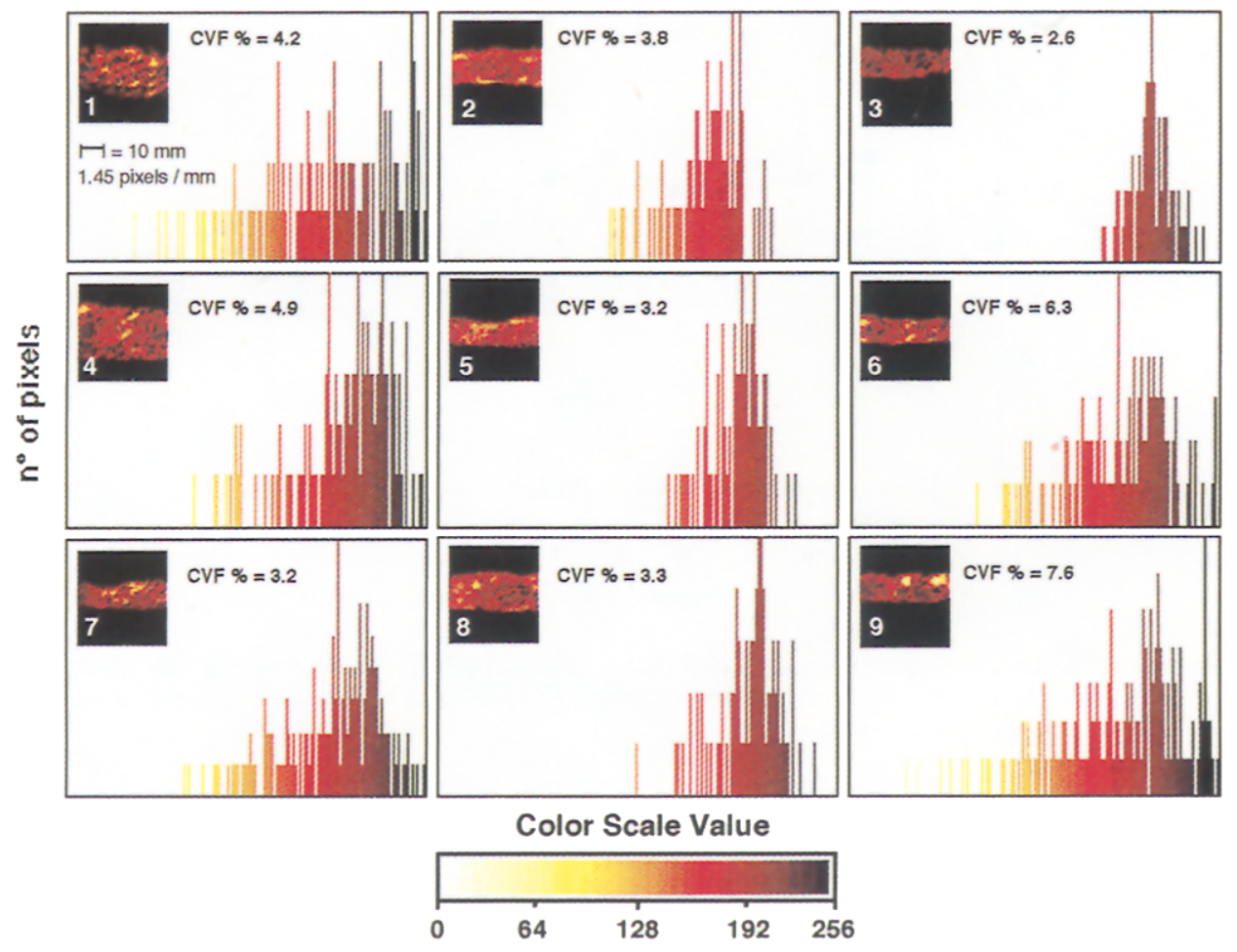

Figure 4 Color scale histogram obtained in all studied patients $(n=9)$. Each panel reports (top left corner) the sample of interventricular septum from which the histogram was derived; the number indicates the studied patient (see Table 1).

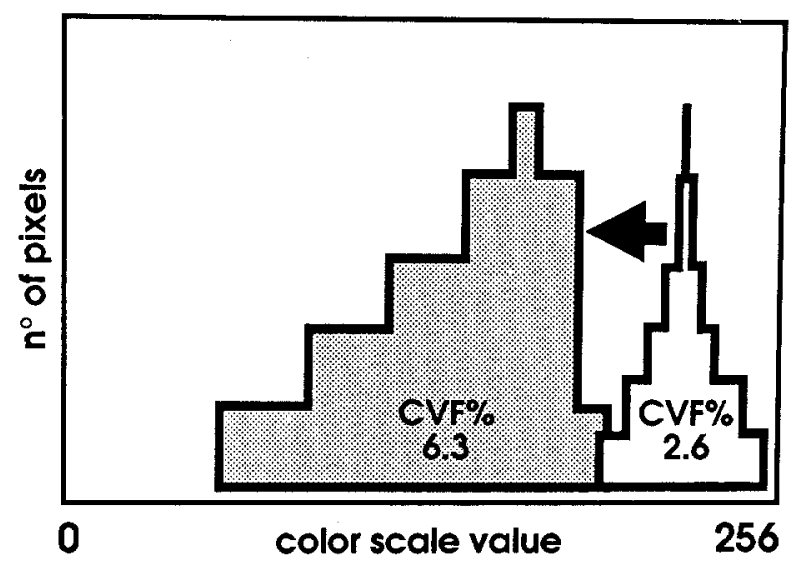

Figure 5 Schematic representation of color histograms derived, respectively, from a patient with a low (empty histogram) and a patient with a high (patterned histogram) collagen volume fraction. Normal CVF values are up to $2 \%$.

Another possible contributing factor is the abnormal left ventricular diastolic filling that is a common finding among patients with arterial hypertension and is probably associated with left ventricular hypertrophy. Diastolic dysfunction is known to affect the cyclic change in tissue reflec-
Table 2 Differences in parameters describing the histogram morphology

\begin{tabular}{lrcc}
\hline & \multicolumn{1}{c}{ Controls } & Hypertensives & p Value \\
\hline $\mathrm{SK}$ & $0.55 \pm 0.12$ & $2.79 \pm 0.57$ & $*$ \\
$\mathrm{~K}$ & $0.58 \pm 0.22$ & $8.39 \pm 3.69$ & $\dagger$ \\
$\mathrm{Bb}$ & $79.29 \pm 6.55$ & $141.89 \pm 52.22$ & $\dagger$ \\
\hline
\end{tabular}

$S K$, Skewness; $K$, kurtosis; $B b$, broad band

${ }^{*} p<0.01$.

$t p<0.001$.

tivity; however, the mechanisms responsible are not yet known. In six patients we have observed an altered diastolic function associated with blunted cyclic changes in echoreflectivity. The changes appear to be closely associated with regional mechanical performance of the tissue. ${ }^{23}$ It is possible that retention of normal systolic-to-diastolic variation of myocardial echoreflectivity might provide a sensitive index of cardiac function.

\section{Clinical Application}

Because myocardial fibrosis is increased in several common types of heart disease, performing noninvasive tissue characterization of myocardial structure to 

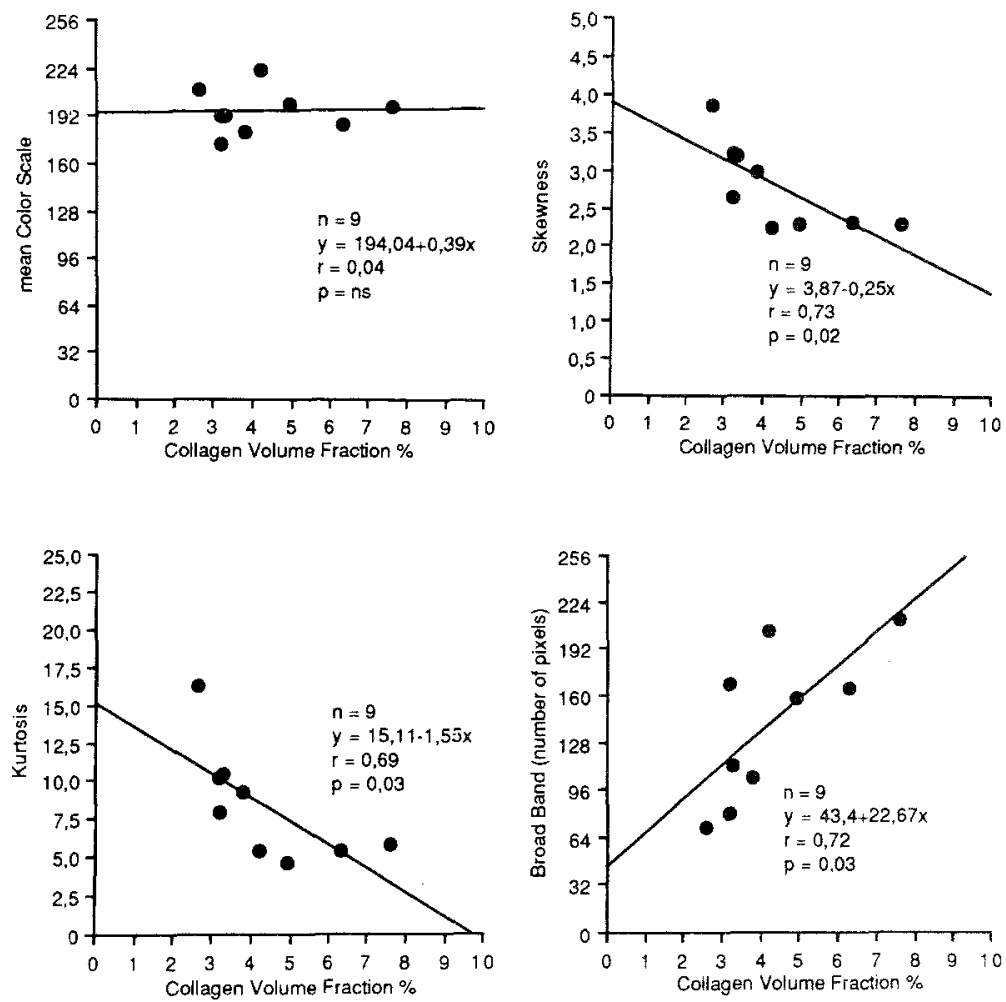

Figure 6 Correlation between collagen volume fraction and mean color scale value (top, left panel), skewness (top, right panel), kurtosis (bottom, left panel), and broad band (bottom, right panel) about the color scale. Values are derived at end diastole; each dot represents one patient.

delineate the extent of collagen accumulation in tissue may play a relevant role in the clinical outcome of these patients, in particular hypertensive patients in whom reversal of left ventricular hypertrophy is considered a desirable goal of therapy.

Despite the increased sensitivity for detection of collagen accumulation in tissue, ${ }^{17}$ the dissemination of these techniques is hampered by the difficulties in setting up a system for ultrasonic tissue characterization. More recently the addition of image-processing capabilities to standard personal computers and the availability of analysis software has provided a lowcost solution to improve image analysis. ${ }^{24,25}$ The technology we used to analyze the video signals is relatively simple and has the advantage that it can be added to commercially available echocardiographic instruments, a fact that greatly increases the clinical applicability of the method. Although backscatter analysis provides a more independent approach from instrumentation settings and from the operator, it is a more complex approach that requires a system not readily available and the potential of the video-processed signal analysis has been shown to reproduce the information of backscatter analysis in many conditions. ${ }^{\mathrm{I}}, 23$

\section{Limitations of the Study}

Some limitations of this study should be acknowledged. As far as the system of analysis is concerned, the video signals we analyze correspond to radio frequency signals that have undergone several processing steps in the echocardiographic instruments to optimize the image. Thus regional alterations of image texture must be interpreted with caution. Furthermore, in a transthoracic approach the effect of the chest wall and depth of cardiac structure from the transducers on tissue imaging is unpredictable.

To ensure a more independent approach from the characteristics of the ultrasound image system and to obtain comparable data between subjects, we have indicized all the values obtained using blood echoreflectivity assessed inside the left ventricle. Other authors used parietal pericardium as an internal standard for setring the gain of the instrumentation. ${ }^{11}$

Another possible source of problems is the anisotropic properties of ultrasounds. ${ }^{26}$ We have studied 
our patients by parasternal long-axis approach. The focus was set and kept perpendicular to the interventricular septum to obtain the same angle of insonification in each subject; by using this approach the sound beam is perpendicular to all three muscular layers of the interventricular septum.

To minimize the sampling variability we have chosen the region for analysis in the same area where the specimen was taken, using as reference the echo images obtained during the biopsy procedure. One absolute limitation to the applicability of our method is that it can be applied only in patients with a good acoustic window.

Finally, there is the concern that endomyocardial biopsy tissue obtained from the interventricular septum may not be representative of the ventricular free wall. Morphometric analysis of septum and free wall in postmortem tissue from hypertensive hearts would suggest the septum is similarly involved and representative. ${ }^{7}$

\section{Conclusions}

In conclusion, tissue characterization with a simple approach that represents a logical extension of present echocardiographic instruments could be practical and useful in the noninvasive assessment of myocardial structure in hypertensive and hypertrophic states. In particular, it might have clinical importance in documenting the extent of collagen accumulation and in assessing methods designed to prevent its appearance or even to cause its regression.

\section{REFERENCES}

1. Perez JE, Miller JG, Barzilai B, et al. Progress in quantitative ultrasonic characterization of myocardium: from the laboratory to the bedside. J Am Soc Echo 1988;1:294-305.

2. Price RR, Jones TB, Goddard J, James AE. Basic concepts of ultrasonic tissue characterization. Radiol Clin North Am $1980 ; 18: 2 \mathrm{I}-30$

3. Miller JG, Perez JE, Sobel BE. Ultrasonic characterization of myocardium. Prog Cardiovasc Dis 1985;27(2):85-110.

4. Marini C, Ghelardini G, Picano E, et al. Effects of coronary blood flow on myocardial gray level amplitude in two dimensional echocardiography: an experimental study. Cardiovasc Res 1993;27:279-83.

5. Sagar KB, Pelc LR, Rhyne TI, Howard J, Warltier DC. Estimation of myocardial infarct size with ultrasonic tissue characterization. Circulation 1991;83:1419-28.

6. Chandrasekaran K, Aylward PE, Fleagle SR, et al. Feasibility of identifying amyloid and hypertrophic cardiomyopathy with the use of computerized quantitative texture analysis of clinical echocardiographic data. J Am Coll Cardiol 1989;13(4):83240.

7. Pearlman ES, Weber KT, Janicki JS, Pietra GG, Fishman AP. Muscle fiber orientation and connective tissue content in the hypertrophied human heart. Lab Invest 1982;46:158-64.
8. Huysman JAN, Vliegen HW, Van der Laarse A, Eulderink F. Changes in nonmyocyte tissue composition associated with pressure overload of hypertrophic human hearts. Pathol Res Pract 1989;184:577-81.

9. Kossoff G, Gossett WJ, Carpenter DA, Jellins J, Dadd MJ. Principles and classification of soft tissues by grey scale echography. Ultrasound Med Biol 1976;2:89-105.

10. Hoyt RM, Skorton DJ, Collins SM, Melton HE. Ultrasonic backscatter and collagen in normal ventricular myocardium. Circulation 1984;69:775-82.

11. Shaw TRD, Logan-Sinclair RB, Surin C, et al. Relation be tween regional echo intensity and myocardial connettive tissue in chronic left ventricular disease. Br Heart J 1984;51:46-53.

12. Devereux RB. Detection of left ventricular hypertrophy by M-mode echocardiography. Anatomic validation, standardization and comparison to othr methods. Hypertension 1987 ; 9(S II):19-26.

13. Thomas JD, Khandheria BK. Digital formatting standards in medical images: a primer for echocardiographers. J Am Soc Echocardiogr 1994;7:100-6.

14. Karson TH, Chandra S, Morehead AJ, Stewart WJ, Nissen SE, Thomas JD. JPEG compression of digital echocardiographic images: impact on image quality. I Am Soc Echocardiogr 1995;8:306-18.

15. Villari B, Campbell SE, Hess OM, Mall G, Weber KT, Krayenbuehl HP. Influence of collagen network on left ventricular systolic and diastolic function in aortic valve disease. J Am Coll Cardiol 1993;22:1477-84.

16. Madras EI, Barzilai B, Pérez JE, Sobel BE, Miller JG. Changes in myocardial backscatter throughout the cardiac cycle. Ultrason Imaging 1983;5:229-34.

17. Picano E, Pelosi G, Marzilli $M$, et al. In vivo quantitative ultrasonic evaluation of myocardial fibrosis in humans. Circulation 1990;81:58-64.

18. Vered Z, Barzilai B, Mohr GA, et al. Quantitative ultrasonic tissue characterization with real-time integrated backscatter imaging in normal human subjects and in patients with dilated cardiomyopathy. Circulation 1987;76:1067-73.

19. Perez JE, Barzilai B, Madras EI, et al. Applicability of ultrasonic tissue characterization for longitudinal assessment and differentiation of calcification and fibrosis in cardiomyopathy. J Am Coll Cardiol 1984;4:88-95.

20. Mimbs JW, O'Donnell M, Bauwens D, Miller JG, Sobel BE. The dependence of ultrasonic attenuation and backscatter on collagen content in dog and rabbit hearts. Circ Res 1980;47: 48-58.

21. Tanaka M, Fujiwara H, Onodera T, Wu D-J, Hamashima $Y$, Kawai C. Quantitative anamysis of myocardial fibrosis in normals, hypertensive hearts and in hypertrophic cardiomyopathy. Br Heart J 1986;55:575-81.

22. Gigli G, Lattanzi F, Lucarini $A R$, et al. Normal utrasonic myocardial reflectivity in hypertensive patients. A tissue characterization study. Hypertension 1993;21:329-34.

23. Olshansky B, Collins SM, Skorton DJ, Prasad NV. Variation of left ventricular myocardial gray level on two-dimensional echocardiograms as a result of cardiac contraction. Circulation 1984;70:972-7.

24. Morris $\mathrm{R}$. Image processing on the Macintosh. IEEE Computer 1990;103-6.

25. Lennard P. Image analysis for all. Nature 1990;347:103-4.

26. Aygen M, Popp RL. Influence of orientation of myocardial fibers on echocardiographic images. Am J Cardiol 1987;45: 248-63. 